\title{
UNA RECREACIÓN QUIJOTESCA EN LA NARRATIVA PERUANA: "HISTORIA DE CIFAR Y DE CAMILO" DE EDGARDO RIVERA MARTÍNEZ
}

\author{
Fernando Rodríguez-Mansilla \\ Hobart and William Smith Colleges \\ Geneva, Nueva York, Estados Unidos \\ mansilla@hws.edu
}

Esta nota analiza el cuento "Historia de Cifar y de Camilo" de Edgardo Rivera Martínez a partir de la intertextualidad que presenta con Don Quijote de la Mancha, a través de la personalidad soñadora del protagonista y el episodio de la estancia en el palacio de los duques (caps. XXX-LVII de la segunda parte de 1615). Igualmente, de forma más o menos explícita, el cuento ofrece conexiones con el medieval Libro del caballero Zifar. "Historia de Cifar y de Camilo" es una rara avis en el panorama de la narrativa peruana y reclama un análisis que reconozca y analice su singularidad en el corpus de relatos sobre adolescentes. De hecho, la dificultad para encajar el relato en alguno de los modelos de escritura usuales en la literatura peruana (narrativa urbana, narrativa sobre adolescentes, narrativa andina) ha llevado, tal vez, a que se le catalogue como "un intento lúdico" (Álamo-Cosigny 170) o experimental, por su ambientación urbana, lejos de la recreación del mundo andino que era característico del autor. Esta singularidad del cuento obedecería, precisamente, al fenómeno de intertextualidad que se explora aquí, el cual puede ayudar a esclarecer el significado final del texto.

En efecto, si bien este cuento se inserta en un corpus ingente de la narrativa peruana, el del adolescente en la ciudad, no encaja en el panorama que trazó Jorge Eslava en su sobresaliente estudio dedicado a este tipo de relato. Para empezar, el cuento, ambientado en Lima, no parece encajar en la obra de Rivera Martínez, a quien se identifica como un exponente de la narrativa andina en la historiografía (Gnutzmann 202). Ocurre que la fecha de composición del texto, 
1980, tampoco corresponde a la periodización, no obstante coherente y bien razonada, que elaboró Eslava: las narraciones, largas o cortas, con adolescentes como protagonistas que se publicaron entre principios de la década de 1970 y 2000 se caracterizan por la presencia de la violencia y el nihilismo como actitud vital; mientras que, entre los años sesenta y setenta, las narraciones desarrollan el retrato del grupo o collera de amigos que descubren la ciudad y ejercen los rituales del machismo (Eslava 117-181). El perfil del protagonista del cuento de Rivera Martínez no se inserta en ninguno de esos paradigmas.

Según Consuelo Álamo-Cosigny, para quien el relato es una novela corta, "no se ve afrontamiento entre las dos clases que están presentes en la ficción, sin embargo, el mensaje principal que se transmite en ella es la urgencia de un cambio de estructuras sociales y económicas más humanas y más justas" (169). Este sesgo interpretativo, que me parece descaminado, proviene, seguramente, de una identificación mecánica de Rivera Martínez como miembro de la Generación del 50, a cuyos narradores se les atribuye la práctica del neorrealismo, una estética que buscaba retratar la ciudad con los problemas sociales y existenciales que la circundan (González Vigil 238-243). El afán de crítica social como objetivo primordial queda claro en escritores contemporáneos como Enrique Congrains Martín, Mario Vargas Llosa u Oswaldo Reynoso, pero en Rivera Martínez conforma solo un elemento de la narración y no exactamente su médula. La crítica social que podría desprenderse del cuento -la notoria desigualdad entre la mansión de Ivonne y la pobreza de la familia de Camilo- se ve muy mellada cuando se consideran dos recursos que me parecen claves para revelar la esmerada construcción de un significado que se centra en la experiencia adolescente de la ensoñación y la búsqueda de un sentido de la propia existencia: la intertextualidad que se presenta con Don Quijote de la Mancha de forma más o menos evidente y, más sutilmente, con el Libro del caballero Zifar; y un estilo que incorpora rasgos lingüísticos castizos, si no identificables al menos afines a la lengua del Siglo de Oro. Ambos recursos provocan un efecto de distanciamiento frente al modo de representación realista típico de la narrativa peruana contemporánea de Rivera Martínez.

Las referencias a Don Quijote y el Libro del caballero Zifar se encuentran en boca del profesor de Camilo, a quien el adolescente pregunta por el nombre, tan extraño, de su amigo felino:

[Cifar] es el nombre de un famoso caballero andante, personaje de una novela de caballerías, como fue también Don Quijote. [...] [Don 
Quijote] es el héroe de una famosa y muy antigua obra, escrita por un autor que se llama Cervantes. Era un señor que se imaginó que eran verdad esas historias y que se echó por el mundo para ayudar a los desvalidos y castigar a los malos, a imitación de los héroes de esas novelas (Rivera Martínez, "Historia" 208).

Si bien el profesor resume bien el afán justiciero de don Quijote, el "quijotismo" que se plasma en el cuento de Rivera Martínez tiene cuño romántico y no consiste tanto en ejercer una vida activa, sino más bien contemplativa: Camilo puede identificarse con la figura del don Quijote decimonónico que se concebía como el tipo universal del idealista en permanente conflicto con la realidad (Close 57), apartando de su imagen los rasgos de humor y sátira literaria que había poseído el texto cervantino en el siglo XVII. Por eso, en el breve resumen del profesor de Camilo no se encuentra rastro alguno de la comicidad inherente al libro durante el Siglo de Oro, sino que se resaltan las virtudes de su protagonista, basadas en la nostalgia por un orden supuestamente perdido, que el enajenado quisiera recuperar a través de la fantasía. Este idealismo quijotesco es comprensible en un personaje como Camilo, un adolescente sensible y soñador que, siendo consciente de los problemas económicos que agobian a su familia, no se revela frente a ellos ni enjuicia a la sociedad que los provoca, sino que se propone la evasión con aquel "corazón de poeta", propio de su edad, que advierte un reseñista reciente (Carhuaricra Anco párr. 2).

Como don Quijote, Camilo es un soñador de extracción humilde (el manchego era un hidalgo pobre y vivía en una aldea), por lo que su fascinación frente al entorno de Cifar no es muy lejana a la que experimenta el protagonista cervantino cuando, en la segunda parte de la novela, es acogido por los duques. Estos últimos eran aristócratas que se divertían a su costa y se aprovechaban de él, sometiéndolo a toda clase de burlas, aunque lo consentían y ofrecían comodidades que nunca había gozado como caballero andante. ¿Qué ocurre en la "Historia de Cifar y de Camilo"? Tras el acuerdo monetario con Ivonne, la dueña de la casona, Camilo pasa tiempo con Cifar y goza de una vida diríase palaciega junto a él, ya que recibe las atenciones de la sirvienta (una mujer de raza negra llamada, irónicamente, Nieves), la cual lo recibe con "té delicioso, y las galletas inglesas y chocolates suizos" (Rivera Martínez, "Historia" 212), en medio de tardes crepusculares barranquinas con vistas al mar. Sin duda, la localización de los hechos en el distrito de Barranco permite trazar un ambiente melancólico y de antemano lírico, considerando 
el prestigio literario que posee este barrio en la literatura peruana a través de la figura de José María Eguren y, sobre todo, de La casa de cartón de Martín Adán, cuyo protagonista adolescente también aprovecha la atmósfera melancólica del barrio que estimula la creatividad literaria (Eslava 200). Dicha atmósfera añade romanticismo a la narrativa y es indesligable de su quijotismo idealizador.

La extravagante oferta de trabajo, consistente en entretener al gato, aparece en el mejor momento, considerando que el padre del adolescente ha perdido el empleo. Incluso, el padre de Camilo lo alienta a que acepte el trabajo en casa de Ivonne y que descarte el de vender cigarrillos en el cine, porque vislumbra que el vínculo laboral con el gato será más duradero. Este periodo dichoso acaba, no obstante, cuando Ivonne y Cifar desaparecen, sin anunciarlo, para hacer un viaje a Estados Unidos. Tras recuperarse de la sorpresa, Camilo se siente defraudado, francamente burlado, considerando que descartó otro trabajo, mucho más convencional y seguro, para estar cerca de Cifar. Ante el chasco, sus padres intentan aleccionarlo con resignación, mientras sus hermanos se mofan de lo que le ha ocurrido. Cualquier ira o resentimiento que pueda albergar Camilo frente a Ivonne en este punto del relato es mitigado por la afirmación de su padre -"Así es la vida de los pobres" (Rivera Martínez, "Historia" 220)-, por la burla de su hermano mayor, que lo llama "idiota" (ibid.), mas no objeta el abandono de Ivonne; y por el orgullo que intenta transmitirle su hermana Cata, quien exclama " $¡$ No seremos nunca más juguetes de nadie!", a lo que añade la idea de que valió la pena haber vivido la experiencia, porque "era como un sueño para nosotros" (Rivera Martínez, "Historia" 221). Tras recibir este respaldo, Camilo se aferra al recuerdo y a intentar consolarse con él. Por todo esto, no es posible extraer del final del relato mensaje alguno de cambio social o de denuncia de desigualdades. Gracias al ambiente narrativo de temple quijotesco romántico que establece el narrador, la desaparición de Ivonne con Cifar y el chasco laboral de Camilo es lo más cercano a una de esas derrotas típicas de don Quijote en las que se golpea de frente con la realidad, pero que no aniquilan en él el deseo de la fantasía. Como el manchego, Camilo no dejará de soñar con la compañía de Cifar y fantasear con toda la belleza que había traído a su vida, aunque fuese por un tiempo tan breve.

Conviene explicar ahora la conexión entre el nombre de Camilo y el de don Quijote, que acaba por cerrar el círculo de correspondencias quijotescas que hemos establecido. Camilo provendría de "Camilote": Camilote era el hidalgo extravagante y enamorado que aparece en el libro de caballerías 
llamado Primaleón (1512). Dámaso Alonso, en un artículo clásico ("El hidalgo Camilote y el hidalgo don Quijote") planteó las semejanzas entre Camilote y don Quijote, partiendo de la semejanza fonética, a la que se suman algunos episodios que los aproximan, especialmente en lo concerniente a cómo ambos son burlados por figuras de autoridad (en el cuento de Rivera Martínez ese sería el rol de Ivonne). Se piensa que Miguel de Cervantes pudo adaptar este personaje del Primaleón o bien de la portuguesa Tragedia de don Duardos, donde también aparece (Alonso 24-28). De Camilote provendría la fealdad del hidalgo manchego y su amor ridículo hacia una mujer igualmente desagradable, pero que en su imaginación es bellísima. Si bien la terminación “-ote" podría explicarse también a partir del personaje de Lanzarote (Riquer 49), resulta convincente la conexión con Camilote, ya que ambos, el manchego y el personaje de Primaleón, son hidalgos risibles e idealistas. A través de la adopción del nombre de Camilo para su protagonista, Rivera Martínez estaría honrando esta genealogía del nombre de don Quijote, haciendo el guiño cervantino algo más sutil todavía.

Queda observar la intertextualidad con el Libro del caballero Zifar, texto del siglo XIV que solo cuenta con una edición impresa en el Siglo de Oro, la sevillana de 1512, cuya lectura no consta en la obra de Cervantes, pero que bien pudo leer Rivera Martínez en alguna edición del siglo XX ${ }^{1}$. En primer lugar, debe observarse el uso del superlativo, que en el cuento de Rivera Martínez cumple la función de significar lo extraordinario, en contraste con la pobreza que rodea a Camilo. Cifar es descrito con superlativos absolutos, como "blanquísimo", "lindísimo" o "el gato más hermoso y engreído de Barranco" (Rivera Martínez, "Historia" 201). En el Zifar y en otros textos caballerescos, incluido Don Quijote de la Mancha, el superlativo es también recurrente. En el Zifar, se dice que el protagonista "seméjame que mejor caballero sea en todo el mundo en armas e en todas buenas costumbres" (102); de un alano, que es "el mejor del mundo" (392); o de un conde, "que era el más anciano e el más poderoso de toda la tierra" (228). Así como el gato Cifar es "un gato cosmopolita" (Rivera Martínez, "Historia” 210) porque es persa, adquirido en

\footnotetext{
Quizás la más popular sea la de la antología Libros de caballerías españoles, publicada en 1960, que incluye el Zifar, el Amadis de Gaula y Tirante el Blanco. En este trabajo, las citas del Libro del caballero Zifar provienen de la edición de Joaquín González Muela que figura en la bibliografía. En adelante, para referirme al personaje caballeresco escribo "Zifar", con ortografía anacrónica que delata su origen medieval, mientras que sigo con el moderno "Cifar" para designar al felino.
} 
París y con muchos viajes encima, el medieval Zifar, como caballero andante, era un peregrino cuyo origen estaba en las Indias orientales y que viajaba por reinos extraños buscando sus aventuras. Por otro lado, el escudero del caballero Zifar empieza siendo conocido simplemente como "el ribaldo", es decir una especie de pícaro, que quedaba caracterizado por vestir como pobre, pues este personaje se refería a sus propios vestidos como "viles" (150), en contraste con los de su amo, quien lo trataba cordialmente como "amigo". Precisamente, la pobreza de Camilo es anotada a través de "la pobreza de mi ropa, mis pies casi descalzos" (Rivera Martínez, "Historia" 203) y él se constituirá en otro escudero o compañero de aventuras del felino.

Más curioso resulta el comentario del profesor de Camilo sobre el protagonista del Zifar, ya que afirma que el caballero era "todo un modelo de sosiego, de prudencia" (Rivera Martínez, "Historia" 208). Este comentario tal vez proviene de la lectura de la sección de los "castigos ['consejos'] del rey de Mentón", que configura toda la tercera parte de la obra (233-322). Dicha sección constituye una colección de ejemplos propios de la literatura sapiencial, en torno a la conducta honorable y el buen gobierno, cercana, en ese sentido, a los consejos de don Quijote a Sancho Panza antes de que este se dirija a su gobernación de la Ínsula Barataria (caps. XLII y XLIII de la segunda parte). Este conocimiento que exhibe el personaje de Zifar es el que puede explicar bien la afirmación del profesor, aunque la mirada lúdica del adolescente Camilo lleve a encontrar bien pronto el lado burlesco del comentario aplicado al gato, que él encuentra más bien juguetón, antítesis de aquel celebrado sosiego del personaje caballeresco.

Otro elemento del Libro del caballero Zifar que bien puede explicar el desenlace del cuento es la aventura de las Islas Dotadas. En la cuarta y última parte de la obra se relatan los "Hechos de Roboán", el hijo del caballero Zifar. Roboán viaja a las Islas Dotadas, donde el caballero se hace emperador y alcanza toda prosperidad. Roboán, instigado por el diablo en disfraz de doncella, le exige a su esposa, la emperatriz, una serie de "donas" u obsequios, que son algunos animales espléndidos, cuya belleza lo hace dichoso como caballero que ejerce la caza. Primero, se trata de un alano blanco, "más blanco que el cristal" (391), luego un azor, descrito como "más albo que la nieve" (395) y, finalmente, un caballo "muy blanco e muy fermoso" (401). Al final, todos los obsequios no eran más que una tentación del diablo que conduce a Roboán a la pérdida de su esposa y del reino, el cual abandona y que queda, a partir de entonces, como un sueño o una aventura más en su pasado hazañoso. La desazón al acabar aquella aventura no está muy lejana 
al final de la fantasía de Camilo en la mansión de Ivonne. Con todo, hay que admitir que el desenlace de este episodio del Zifar, que encierra una burla urdida por el demonio, es intercambiable con el del episodio de los duques en Don Quijote de la Mancha. De todas formas, ambos finales ayudan a comprender el final mismo de la "Historia de Cifar y de Camilo" en términos de aventura caballeresca (seria en el Zifar, cómica en Don Quijote) antes que como crítica al orden social.

Por último, para acabar con el Libro del caballero Zifar, un elemento de posible intertextualidad, algo más remoto, es el siguiente: el caballero Zifar perdía un caballo cada diez días. En el cuento, Cifar se encuentra varias veces con Camilo, quien reaparece en su vida por tres razones muy diversas: primero, por un encuentro azaroso; luego por un plan organizado por su hermana para verlo y para robárselo; después, convocado por Ivonne. De esa forma, parece que Cifar pierde y recupera a Camilo, tal como le ocurría al caballero que cambiaba de montura periódicamente.

Ahora bien, resulta llamativo este fenómeno intertextual, tanto en lo que concierne a Don Quijote como al mismo Zifar, considerando que las obras referidas son clásicas (una del siglo XIV y la otra del siglo XVII) y algo distantes (tanto en lo formal como en lo ideológico) de la agenda de la narrativa peruana de la segunda mitad del siglo XX, cuyos exponentes, por lo general, mostraban una mayoritaria inclinación por autores latinoamericanos, norteamericanos y europeos, y prácticamente nulo interés en la literatura peninsular. Tan solo considérese que, de los catorce autores, todos contemporáneos de Rivera Martínez, encuestados por Abelardo Oquendo en su antología Narrativa peruana 1950/1970, apenas Carlos Eduardo Zavaleta menciona a Miguel de Cervantes y Mario Vargas Llosa habla, exquisitamente, de Joanot Martorell, el autor de Tirante el Blanco (Oquendo 48-50). De hecho, las recreaciones del personaje de don Quijote en la narrativa peruana del siglo XX previas al cuento de Rivera Martínez son muy pocas. Además de la curiosa novela Don Quijote en Yanquilandia de Juan Manuel Polar (1925), apenas se cuenta con dos cuentos modernistas que ha estudiado Moisés Sánchez Franco. En su análisis, este último detecta una actitud de desmitificación del personaje, debido al interés del modernismo de fundar su propia tradición y marcar distancia de lo que significaba cualquier herencia hispana.

En dicho contexto literario, una recuperación de aquellas obras como la que lleva a cabo Rivera Martínez en su relato solo es posible gracias a una relación mucho más amistosa y creativa, que se empieza a desarrollar entre los miembros de la Generación del 50, sobre todo en la lírica, a través de poetas 
como Jorge Eduardo Eielson, Carlos Germán Belli y Javier Sologuren, según lo ha analizado Solís Mendoza. Aunque no se le suele considerar miembro de esta generación, seguramente la última más eminente de las letras peruanas, se comprende que Rivera Martínez cultivara un conocimiento del Siglo de Oro no solo por un interés personal, sino también porque pudo recibir el estímulo de las clases de Luis Jaime Cisneros en la facultad de Letras de la Universidad de San Marcos en la década de 1950 (Ferreira 18). Este último, que había vuelto poco tiempo atrás de la Argentina, importó al Perú la estilística y con ella un currículo de estudios literarios que, a través de una perspectiva novedosa, ponía énfasis en los grandes autores españoles de los siglos XVI y $\mathrm{XVII}{ }^{2}$. Precisamente uno de los representantes de la estilística como método de análisis era Dámaso Alonso, a quien se le debe la identificación de don Quijote con el hidalgo Camilote del libro Primaleón.

En lo que respecta al estilo en el que está compuesto el relato, se observan ciertos rasgos castizos o al menos que aspiran a distinguirse de la norma lingüística peruana contemporánea. Para empezar, el título del texto evoca los que solían llevar los libros de caballerías del Siglo de Oro, los cuales en sus títulos solían alternar entre "libro" e "historia", vocablos que expresaban su carácter narrativo (pues "novela" solo designaba por entonces a las novelas cortas) y se ceñían al criterio, clásico, de emplear el nombre del protagonista para nombrar la obra. En torno al Zifar, consta que en los inventarios de bibliotecas se emplea el rótulo de "historia" para denominarlo: una "Historia del caballero Cifar" aparece en el catálogo de la librería del conde de Gondomar, a inicios del siglo XVII (Díez Borque 197). De la mano de este indicio, convendría reparar en el hecho de que en el título del cuento se repite la preposición "de", cuando el contexto no lo requiere (pues no se encuentra ambigüedad recusada que necesite evitarse). En la lengua del Siglo de Oro, existen ejemplos mixtos, tanto de dicha repetición como de elipsis, por lo que no puede considerarse, estrictamente, un rasgo clásico (Keniston 41.22). No obstante, se produce una rarificación del lenguaje para generar un efecto literario de distanciamiento, que refuerza el significado de fantasía e imaginación lírica que motiva al protagonista. Lo mismo ocurre con el uso del pronombre "yo" en primer lugar de una frase nominal coordinada, del cual el cuento ofrece dos casos: "Yo y mi hermana Cata" (Rivera Martínez, "Historia" 
201) y "yo y él jugaremos" (Rivera Martínez, "Historia" 211). Se trata de un uso que se encuentra en Cervantes y que obedece a la aplicación del orden natural de los nombres, de acuerdo con la Gramática castellana de Antonio de Nebrija. Así, en los Trabajos de Persiles y Sigismunda, un personaje habla de "yo y mi hermana" (226)3. O en Don Quijote de la Mancha: "Que yo y mi marido nos saldremos de nuestro aposento" (I, XLII, 440). Por último, algo parecido ocurre con la frase "miramos por un buen espacio" (Rivera Martínez, "Historia" 206), donde "espacio" en el sentido de "un buen rato", resulta anacrónico en el español peruano de finales del siglo XX. Su sentido se aclara cuando se recuerda que, en el siglo XVII, espacio "también sinifica el intervalo del tiempo", según Covarrubias en el Tesoro de la lengua castellana o española (827). Estos elementos de estilo (el título del cuento, el orden de los pronombres y el significado de "espacio"), desperdigados a lo largo de la narración, cooperan a establecer una atmósfera que, sin desprenderse de una representación realista, la atenúan y conducen a una dulcificación que se suma a la que ejerce la mirada soñadora del protagonista.

Considerando el análisis de los materiales aportados, es posible sostener que "Historia de Cifar y de Camilo" se encuentra lejos de una representación estrictamente realista que enfatice la crítica social. Sin embargo, se ha insistido en este supuesto interés de denuncia. Según Álamo-Cosigny, la palabra clave que sintetiza el texto es la ausencia:

El factor ausencia es el que moviliza todas las fuerzas del relato. La ausencia de dinero en la casa de los dos niños, Camilo y Cata, hace que estos se desempeñen como vendedores ambulantes, que Cata teja botines en lana para venderlos, o que Camilo acepte cuidar a Cifar, el gato de la familia rica. Más tarde, la ausencia del gato dejará a Camilo sin compañero, sin empleo y, en consecuencia, sin dinero (Álamo-Cosigny 169-170).

Esta interpretación no es del todo exacta, si recordamos que la relación de Camilo con Cifar empezó mucho antes de cualquier tipo de arreglo económico entre él e Ivonne. Sin duda, la coyuntura le exige ganar dinero y

Remito a la nota filológica de Carlos Romero Muñoz al pasaje de la edición citada para una explicación de este uso del pronombre "yo" en primer lugar, que solo empezó a cambiar, por cortesía, en la época del mismo Cervantes, aunque este prefería la forma más tradicional. 
que la dueña de la casa se lo ofrezca para que pase tiempo con el gato resulta muy conveniente para Camilo, pero su vínculo con Cifar estaba ya cimentado y el mismo Camilo traza una línea divisoria clara entre el gato y su entorno, que al adolescente le resulta repulsivo:

Pensé con rencor en el lujoso y siempre cerrado portal del salón, en los cristales y la platería del comedor, en ese auto tan relumbrante. Detesté aún más los pálidos ojos de la propietaria. Ni siquiera Nieves, generosa como era, y sin duda tan pobre como nosotros, se sustrajo a esa hostilidad. Solo quedó al margen Cifar, no obstante su condición de animal de lujo. Y es que Cifar era para mí un ser absolutamente singular (Rivera Martínez, "Historia” 214).

Así, si las primeras líneas delatan un evidente resentimiento social frente al mundo de los privilegiados frente a los proletarios, las últimas, dedicadas a Cifar, permiten encontrar en Camilo una tendencia constante hacia la fantasía que recorre todo el texto y supera cualquier pensamiento que conduzca a algún tipo de denuncia social que pueda desprenderse de la lectura. Camilo acepta la condición privilegiada de Cifar y la abraza sin problemas, porque para él se trata de algo accesorio. Explico por qué. Frente al factor "ausencia" que proponía Álamo-Consigny, me inclinaría por proponer el de "deseo", que me parece más productivo para el análisis de este cuento en particular. Ciertamente, la ausencia está presente en el entorno de Camilo y varios miembros de su familia se guían por este concepto, ya que en ellos impera el sentido práctico; como su hermana, Cata, quien proponía robarse al gato para apropiarse de algo de esa magia y elegancia de la casona, como si soñara con provocar envidia a las vecinas por ese gato "tan pituco" (Rivera Martínez, "Historia" 205). Sin embargo, para el adolescente el deseo es el que describe mejor sus acciones.

La fascinación de Camilo por el gato proviene de su carácter extraordinario, pero el chico no encuentra en el felino el valor de una posesión que generase envidia en los otros. Según él, Cifar es "tan blanco, tan irreal, tan hermoso" (Rivera Martínez, "Historia" 202). Encuentra en Cifar un espejo en el cual reflejarse. Su deseo se encuentra encarnado en el felino: una vida contemplativa, propia de un poeta, admirando el mar, que simboliza la belleza de lo insondable. Lo mismo ocurre con la personalidad enigmática que identifica Camilo en el gato, hasta llegar a pensar que Cifar puede soñar con él: “¿Cómo podía ser que Cifar nos extrañara? ¿Se había aficionado tanto a nosotros en una hora de 
juego? ¿Acaso los gatos son cariñosos? ¿Habría soñado conmigo?” (Rivera Martínez, "Historia" 209). Son preguntas que se plantean y nunca tendrán respuesta, por lo que reafirmarán su deseo de estar con Cifar; figurativamente, ser como él. El deseo como móvil de las acciones de Camilo lleva a identificar a Cifar como su particular mediador, en la medida en que identifica la vida del felino como aquella a la que aspira. El deseo y el mediador son términos de la teoría del deseo triangular (todo deseo está mediatizado por un tercero, el mediador) que desarrolló René Girard en el primer capítulo de Mentira romántica y verdad novelesca (9-52).

Nuevamente, se trata de otro guiño cervantino: los sueños caballerescos del hidalgo Quijano provienen de su admiración por Amadís de Gaula; Camilo encuentra en su "amigo" Cifar un modelo vital. Si, como Camilo sostiene, consideró al felino su amigo desde el inicio (Rivera Martínez, "Historia" 203), resulta conveniente recordar que el amigo es "el otro yo", como decía Cicerón. Como explica bien Jorge Eslava, el adolescente está permanentemente abocado a una búsqueda existencial que lo lleva a tomar distancia de su familia y encontrar su propio camino. Dicha búsqueda la lleva a cabo el adolescente en compañía de sus pares (Eslava 45). En este caso, el protagonista había hallado en el gato Cifar a ese compañero con el que asumir roles y abrazar aquella introspección típica de la adolescencia.

Este deseo que promueve la identificación con Cifar no tiene nada que ver con el dinero o el status social, evidentemente privilegiado, que lo rodea. Si Ivonne mantiene a Cifar como un objeto precioso, como una joya en una urna, que es su mansión barranquina, Camilo deja claro bien pronto que a él no le atrae nada de eso, sino la presencia del gato y el tipo de vida (sosegada, contemplativa) que él representa, que es lo más cercano a sus propias inclinaciones de soñador o "poeta", como lo juzgan algunos (Rivera Martínez, "Historia" 204, 216). Camilo incluso emplea términos como "rencor", detestar" y "hostilidad" para dejar en claro que todo lo que rodea a Cifar le repugna, en tanto este último "era para mí un ser absolutamente singular, con el oro terso de sus ojos, su blancura, su exótica procedencia. Y singular, sobre todo, en su don de suscitar siempre esa sensación de levedad, de elegancia, de alegría" (Rivera Martínez, "Historia" 214). La forma en que se describe a Cifar se parece a la que merece, a menudo, la descripción de la poesía como experiencia. Con la identidad de "poeta" que le atribuyen, la cual le complace, Camilo encuentra en el felino un ejemplo vivo de la poesía, es decir de la belleza que busca para hacer su vida plena. Esta imagen esotérica del gato, que encarna conceptos como silencio, serenidad, misterio y belleza 
sofisticada, fue desarrollada por Charles Baudelaire (Van Vechten 321) y ha pervivido desde el romanticismo hasta nuestros días. Rivera Martínez ha dado testimonio de su conocimiento de esta tradición en una nota titulada "Gatos literarios". Allí, mencionando el cuento que nos ocupa, confirma que Cifar es un "gato elegante, onírico, pero también y a su modo afectuoso. Uno que de alguna manera simboliza una vida tersa y diáfana, pero en más de un sentido secreta" (135).

Finalmente, el significado del cuento de Rivera Martínez se sostiene grandemente en la caracterización, de origen romántico, del gato como un ser enigmático, distinguido e intrínsecamente bello, como ya se dijo. La blancura de Cifar, en ese sentido, resalta su singularidad, su condición de criatura extraordinaria, en un contexto, como el de Camilo y su familia, que está marcado por lo cotidiano y por la asociación, tradicional, de pobreza y suciedad. Si bien el gato no alcanza a ser un símbolo de la soledad de Ivonne (que hubiera sido lo presumible), los personajes a su alrededor tienden a infantilizarlo, por lo que, junto a Camilo, Cifar se asemeja a un pobre niño rico. Ivonne lo trata como una posesión, lo cual la hace más antipática de lo que ya es por la manera en que trata a Camilo y su hermana. Lo que para Camilo es la mayor virtud y belleza de Cifar, su silencio y aparente indiferencia frente al mundo (como si estuviera por encima de todo), es al mismo tiempo lo que provoca en Ivonne y todos los que viven en la casa su mayor intriga y desasosiego, ya que la vida de la casona parece girar alrededor del gato. Finalmente, la sutil ridiculización que emprende el narrador del cuento de los personajes de la casona y de todos aquellos que desvalorizan su relación con el gato, es la que hace que el lector acabe por abrazar el hechizo que Cifar ejerce sobre Camilo y convertirlo en una imagen memorable de la fantasía de un adolescente idealista.

La fantasía es un ingrediente que, en obras posteriores más ambiciosas, Rivera Martínez sigue vinculando con referencias al género de las caballerías, que dotan a su narrativa de una dimensión mágica, como ocurre en el Libro del amor y de las profecías (1999) -nótese la presencia del "de" repetido otra vez-, donde un personaje llamado Urganda evoca a la famosa Urganda La Desconocida del Amadís de Gaula. Más provocador resulta el personaje de Justina, el amor más bien carnal del protagonista Esteban. Mientras Luis Nieto Degregori especula que allí se podría encerrar una alusión al personaje de la Justina del legendario cuento "Warma Kuyay" de José María Arguedas (232), un lector de Siglo de Oro postularía, en cambio, a la protagonista de La pícara Justina (1604), muchacha que se caracterizaba precisamente por 
su ligereza de cascos. Naturalmente, esto último es solo una intuición y merecería explorarse más. Sin embargo, sería una señal más de la necesidad de considerar otras tradiciones literarias, no solo las estrictamente andinas, o por extensión peruanas, para examinar a profundidad la obra, tan rica y sugerente, de Edgardo Rivera Martínez y ponderar su originalidad. El análisis de "Historia de Cifar y de Camilo" que se ha ofrecido aquí es tan solo una invitación a hacerlo.

\section{BIBLIOGRAFÍA}

Álamo-Cosigny, Consuelo. "El relato breve de Edgardo Rivera Martínez". De lo andino a lo universal: la obra de Edgardo Rivera Martínez. César Ferreira e Ismael P. Márquez (eds.), Lima, Fondo Editorial de la Pontificia Universidad Católica del Perú, 1999, pp. 155-178.

Alonso, DÁmaso. "El hidalgo Camilote y el hidalgo don Quijote". Del Siglo de Oro a este siglo de siglas (notas y artículos a través de 350 años de letras españolas). Dámaso Alonso, Madrid, Gredos, 1968, pp. 20-28.

Carhuaricra Anco, Miguel Ángel. "Historia de Cifar y de Camilo de Edgardo Rivera Martínez: la revelación del alma infantil". Red Literaria Peruana (web), 19 de julio de 2018, consultado el 21 de noviembre de 2021, disponible en: https://redliterariaperuana. com/2018/07/19/historia-de-cifar-y-de-camilo-de-edgardo-rivera-martinez-la-revelaciondel-alma-infantil-por-miguel-angel-carhuaricra/

Cervantes, Miguel De. Don Quijote de la Mancha. Francisco Rico (ed.), Madrid, Real Academia Española / Asociación de Academias de la Lengua Española, 2004.

Los trabajos de Persiles y Sigismunda. Carlos Romero Muñoz (ed.), Madrid, Cátedra, 1997.

Close, Anthony. The Romantic Approach to Don Quixote. A Critical History of the Romantic Tradition in "Quixote" Criticism. Cambridge, Cambridge University Press, 1977.

Covarrubias, Sebastián de. Tesoro de la lengua castellana o española. Ignacio Arellano y Rafael Zafra (eds.), Madrid/Frankfurt Am Main, Iberoamericana/Vervuert, 2006.

Díez Borque, José María. "Bibliotecas y novela en el Siglo de Oro". Hispanic Review 75, $\mathrm{N}^{\circ} 2,2007$, pp. 181-203.

Eslava, Jorge. Adolescentes en la ciudad. Una visión de la narrativa peruana del siglo XX.

Lima, Fondo Editorial de la Universidad Católica Sedes Sapientiae, 2008.

Ferreira, César. "Presentación: perfil de Edgardo Rivera Martínez". De lo andino a lo universal: la obra de Edgardo Rivera Martínez. César Ferreira e Ismael P. Márquez (eds.), Lima, Fondo Editorial de la Pontificia Universidad Católica del Perú, 1999, pp. 17-21.

GIRARD, RenÉ. Mentira romántica y verdad novelesca. Barcelona, Anagrama, 1985. 
GnUtzmann, Rita. "Una retrospectiva sobre medio siglo de narrativa peruana". América sin nombre 13-14, 2009, pp. 194-204.

GonzÁlez Vigil, Ricardo. "La narrativa peruana después de 1950”. Lexis 8, N², 1984, pp. 227-248.

Keniston, Hayward. The Syntax of Castilian Prose. The Sixteenth Century. Chicago, The Chicago University Press, 1937.

Libro del caballero Zifar. Joaquín González Muela (ed.), Madrid, Castalia, 1982.

Libros de caballerías españoles. Felicidad Buendía (ed.), Madrid, Aguilar, 1960.

Nieto Degregori, Luis. "Suma cósmica. Libro del amor y de las profecías de Edgardo Rivera

Martínez". Edgardo Rivera Martínez. Nuevas lecturas. César Ferreira (ed.), Lima, Fondo Editorial de la Universidad Nacional Mayor de San Marcos, 2006, pp. 231-233.

Oquendo, ABelardo. "Encuesta a los narradores". Narrativa peruana 1950/1970. Abelardo Oquendo (ed.), Madrid, Alianza, 1973, pp. 11-49.

RiQuer, Martín De. Aproximación al Quijote. Madrid, Salvat Editores, 1970.

Rivera Martínez, Edgardo. "Historia de Cifar y de Camilo". Cuentos completos. Edgardo Rivera Martínez, Lima, Alfaguara, 1999, pp. 201-221.

“Gatos literarios". Estampas de ocio, buen humor y reflexión. Edgardo Rivera Martínez, Lima, Fondo Editorial de la Universidad Nacional Mayor de San Marcos, 2003, pp. 131-135.

Sánchez Franco, Moisés. "El Quijote en dos cuentos del modernismo peruano". El mar no es ancho ni ajeno. Complicidades transatlánticas entre Perú y España. Ángel Esteban y Agustín Prado Alvarado (eds.), Madrid/Frankfurt Am Main, Iberoamericana/Vervuert, 2019, pp. 69-80.

Solís Mendoza, Nazareth. "Pasión por la influencia. El Siglo de Oro en la poesía de la generación del 50". El mar no es ancho ni ajeno. Complicidades transatlánticas entre Perú y España. Ángel Esteban y Agustín Prado Alvarado (eds.), Madrid/Frankfurt Am Main, Iberoamericana/Vervuert, 2019, pp. 81-99.

Van Vechten, Carl. The Tiger in the House. A Cultural History of the Cat. Nueva York, New York Review of Books, 2007.

Vargas Llosa, Mario. El pez en el agua. Barcelona, Seix Barral, 1993. 